\title{
The Influence of Public Goods on the Development of China's Regional Economy - A Spatial Econometric Approach
}

\author{
Jialin Li \\ SHU-UTS SILC Business School, Shanghai University, China
}

Doi: 10.19044/esj.2018.v14n13p1 URL:http://dx.doi.org/10.19044/esj.2018.v14n13p1

\begin{abstract}
China's economy has been soaring in the recent decades. Nevertheless, it is characterized by problems of imbalance of economic development, which could hinder the sustainable development of the economy. This paper selects data of various kinds of public goods provision and regional economic development status in China from 2007 to 2014, and investigates the influence of public goods on the development of China's regional economy from using a spatial econometric approach. Empirical findings show that there is a significant spatial correlation within the data of public goods investment during 2007 and 2014, and that the investment on public goods has positive influence on development of regional economy. The finding also shows that there exist spatial spillover effects, which means that the investment in regional public goods can boost the economic growth of surrounding regions.
\end{abstract}

Keywords: Public Goods; Regional Economy; Spatial Econometric Models

\section{Introduction}

In 2014, China's national statistical yearbook survey shows that Beijing's per capita GDP reached 99 thousand yuan, while the average GDP of Yunnan, Guizhou, Sichuan and Tibet was below 30 thousand yuan in the same period. The difference was three times. It can be seen that although the speed of China's economic development is far ahead in the world, the imbalance of economic development is also a serious problem, which is a major obstacle to the sustainable growth of China's overall economy. The defects of the current supply system of public goods in China may be one of the most important reasons for this problem. Economic growth is closely related to the construction of public goods investment. For example, in recent years, many major activities in China have stimulated the investment and construction of public goods, and also stimulated economic growth. The 2008 Beijing Olympic Games not only increased the number of public goods, but 
also enabled China to maintain a high-speed economic growth in the 2008 financial crisis. The 2012 Shanghai World Expo also played a similar role. Although the investment and supply of public goods cannot be regarded as the only factor that accelerated the growth of China's economy at that time, it somehow shows that the investment and construction of public goods have played an important role in economic development.

The investment and construction of public goods promote the economic development mainly from two aspects. On one hand, public goods can be used as a direct investment in improving economic returns ( $\mathrm{Li}$ and $\mathrm{Liu}$, 2012). On the other hand, the provision of public goods cannot only promote the effective growth of the labor force and improve the human capital stock, but also stimulate private investment, including capital injection, to indirectly drive regional economic growth (Lucas, 1988). The contribution of different public goods to the regional economy has a size. Duffy-Deno (1998) studied the difference between the promoting effects of different public goods on regional economy. For example, the correlation coefficient of the impact of investment in highway construction and drainage construction on the regional economy was up to 0.3 , while that of water supply construction investment was only 0.07 . In addition, the investment and construction of public goods can drive the economic development of the surrounding areas, namely the spatial spillover effects (Liu, 2010, Luo and Liu, 2015).

But not all the investment and construction of public goods have a positive impact on the economy. Most previous research finds that the effects of public expenditure on consumption and public expenditure on production on economic growth are different. The positive influence of economic growth is mainly from public expenditure on production, while the effect of public expenditure on consumption on economic growth is very difficult to judge. Both Arrow and Kurz (1970) and Aschauer (1989) argued that increasing public expenditure on production can lead to economic growth by increasing the stock of capital. Boldeanu and Ion (2015) reckoned that non-productive fiscal expenditure has a negative impact on per capita GDP. Butkiewicz and Yanikkaya (2011) showed that public expenditure on consumption of the government is harmful to economic growth because of the existence of inefficient government in developing countries. In addition, the expansion of education, health care and other social public goods expenditure can improve people's living conditions, future expectations and consumption to stimulate economic development (Liu, 2013).

In recent years, Chinese government has continuously strengthened the construction of public goods, but the development of public goods in different regions is different. In that way, can the economic development of a region and even the surrounding areas be promoted by strengthening investment and construction of public goods in economically backward areas? Can the 
regional development imbalance be narrowed in the same way? In addition, China has vast territory and different regional economic development. The resources of public goods may also be different at the present stage. Thus, can the same public goods investment and supply bring different degrees of influence to different regions?

Based on data of Chinese 31 provinces from 2007 to 2014, this paper uses the Solow growth model to model the development of the regional economy. It empirically tests the effects of various variables, including the number of people who have received higher education, investment in fixed assets, the number of patents, and investment in research and development (R\&D). Moreover, the paper also uses spatial measurement methods to study the spatial spillover effects of public goods on regional economic growth on the base of the construction of public goods, the degree of economic development and geographic information in various regions. In this paper, public goods are subdivided into 12 items in order to study the impact of different aspects of expenditures on public goods on the economic development and the impact on the surrounding areas. At the same time, the education population, fixed assets investment and the number of patents are used as variables to explore the role of different types of public goods in promoting different aspects of regional economic development. In addition, this paper also conducts regression analysis of the impact of each type of public goods on the economic development of different regions to reflect the differences between regions. Empirical results of this paper contribute to existing literature on choosing the direction of public goods investment.

\section{The Model}

Initially, public goods are included in the Solow growth model, in which economic growth can be expressed as the following form:

$$
Y_{i t}=\mathrm{F}\left(G_{i t}, K_{i t}, L_{i t}\right) \text {, }
$$

in which i and t represent area and time, respectively. $Y_{i t}$ is the GDP of area $\mathrm{i}$ in year t. $Y_{i t}$ can be explained by $G_{i t}$ (public goods investment in area i in year t), $K_{i t}$ (the whole society investment in area i in year $\mathrm{t}$ ) and $L_{i t}$ (the number of effective labor of area i in year $t$ ). The specification of the Solow growth model follows a Cobb-Douglas function,

$$
Y_{i t}=G_{i t}^{1-\beta} K_{i t}^{\beta} L_{i t}^{1-\beta}
$$

in which $0<\beta<1$. In order to eliminate the impact of population differences between regions, this paper uses per capita capital to replace the total amount of capital in Equation (2), and transforms the production function into the following form:

$$
y_{i t}=g_{i t}^{1-\beta} k_{i t}^{\beta} \text {. }
$$


Then the Cobb-Douglas production function is modified to establish a general panel data measurement model, and the formula is as follows:

$$
\ln y_{i t}=\beta_{0}+\beta_{1} \operatorname{lng} g_{i t}+\beta_{2} \ln k_{i t}+\varepsilon_{i t} \text {. }
$$

In order to embody the difference between regions, this paper continues to establish spatial regression model to analyze the effect of public goods investment on regional economic growth taking into account of the spatial spillover effect. Equation (4) is transformed into a spatial econometric model, and the expression is as follows.

$$
\begin{gathered}
\ln _{i t}=\alpha+\tau \ln y_{i t-1}+\rho \sum_{j=1}^{n} W_{i j} \ln y_{j t}+\sum_{K=1}^{K} X_{i t K} \beta_{K}+\sum_{K=1}^{K} \sum_{j=1}^{n} W_{i j} X_{j t K} \theta_{K} \\
+\mu_{i}+\gamma_{i}+\varphi_{i t} \\
\varphi_{i t}=\lambda \sum_{\mathrm{j}=1}^{\mathrm{n}} \mathrm{m}_{\mathrm{ij}} \varphi_{\mathrm{it}}+\varepsilon_{\mathrm{it}}, \mathrm{i} \in\{1,2, \ldots, \mathrm{n}\} \mathrm{t} \in\{1,2, \ldots, \mathrm{T}\}
\end{gathered}
$$

in which $W_{i t}$ is the corresponding value of the $\mathrm{i}, \mathrm{j}$ column in the spatial weight matrix; $y_{i t-1}$ is the lag term of $y_{i t} ; \theta_{K}$ is a spatial lag variable.

After the transformation of the standard formula, four spatial regression models are obtained respectively.

When $\lambda=0$ and the other coefficients are not zero, the Spatial Durbin Model (SDM) is obtained; $\rho=\theta=0$ while $\lambda \neq 0$, the Spatial Error Model is obtained (SEM);

When $\rho=\theta=\lambda=0$, the Spatial Lag Model is obtained;

When $\theta=0$ and the other coefficients are not zero, the Spatial Autocorrelation Model (SAC) is obtained.

To continue the simplification, the first order partial derivative of the explanatory variable $\mathrm{X}$ is carried out as follows.

$$
\left[\frac{\partial Y}{\partial \mathrm{x}_{\mathrm{nK}}}\right]=(1-\rho W)^{-1}\left[\begin{array}{cccc}
\beta_{K} & W_{12} \theta_{K} & \cdots & W_{1 n} \theta_{K} \\
W_{21} \theta_{K} & \beta_{K} & \cdots & W_{2 n} \theta_{K} \\
\vdots & \vdots & \ddots & \vdots \\
W_{n 1} \theta_{K} & W_{n 2} \theta_{K} & \cdots & \beta_{K}
\end{array}\right]
$$

Then according to the above mentioned methods, the SDM, SEM, SAR, and SAC models are modeled separately.

Substituting in $\lambda=0$, the SDM model is:

$$
(1-\rho W)^{-1}\left[\begin{array}{cccc}
\beta_{K} & W_{12} \theta_{K} & \cdots & W_{1 n} \theta_{K} \\
W_{21} \theta_{K} & \beta_{K} & \cdots & W_{2 n} \theta_{K} \\
\vdots & \vdots & \ddots & \vdots \\
W_{n 1} \theta_{K} & W_{n 2} \theta_{K} & \cdots & \beta_{K}
\end{array}\right]
$$

Substituting in $\rho=\theta=0$, the SEM model is:

$$
\left[\begin{array}{cccc}
\beta_{K} & 0 & \cdots & 0 \\
0 & \beta_{K} & \cdots & 0 \\
\vdots & \vdots & \ddots & \vdots \\
0 & 0 & \cdots & \beta_{K}
\end{array}\right]
$$


Substituting in $\theta=0$ or $\lambda=\theta=0$, the SAR and SAC models can be obtained:

$$
(1-\rho W)^{-1}\left[\begin{array}{cccc}
\beta_{K} & 0 & \cdots & 0 \\
0 & \beta_{K} & \cdots & 0 \\
\vdots & \vdots & \ddots & \vdots \\
0 & 0 & \cdots & \beta_{K}
\end{array}\right]
$$

In terms of economic development, this paper divides 31 Chinese provinces into three regions, i.e., East, Centre, and West. The more developed eastern coastal region includes Beijing, Tianjin, Hebei, Liaoning, Shanghai, Jiangsu, Zhejiang, Fujian, Shandong, Guangdong, Guangxi and Hainan. The central region includes Inner Mongolia, Jilin, Heilongjiang, Anhui, Jiangxi, Henan, Hebei and Hunan. The relatively underdeveloped western region includes Shanxi, Chongqing, Sichuan, Guizhou, Yunnan, Tibet, Shaanxi, Gansu, Qinghai, Ningxia and Xinjiang. The longitude and latitude of all 31 provinces are obtained to construct spatial matrix. Moreover, this paper selects data from 2007 to 2014. The data are gathered from the statistical yearbook of the National Bureau of statistics of People's Republic of China. In the data, R\&D investment per capita in 2007 and the educated population in all regions in 2010 are missing. The two data are the virtual data obtained by fitting the data from the previous and the next two years.

The explained variable is the degree of regional economic development. For the measurement of the degree of regional economic development, this paper selects the per capita GDP of the region (unit: 10,000 yuan). The main explanatory variables are per capita expenditure on public goods, as well as a series of fiscal per capita expenditure on public goods, including national defense, public security, education, science and technology, culture, sports and media, social security and employment, health care, environmental protection, urban and rural community affairs, forestry and water affairs and transportation. All the above units are 10,000 yuan. According to the Solow growth model $\mathrm{Y}(\mathrm{t})=\mathrm{F}(\mathrm{K}(\mathrm{t}), \mathrm{A}(\mathrm{t}), \mathrm{L}(\mathrm{t}))$, namely the macro economic growth depends on the amount of assets of $\mathrm{K}(\mathrm{t})$, knowledge and technology $\mathrm{A}(\mathrm{t})$, and effective labor $\mathrm{L}(\mathrm{t})$. Thus, this paper selects per capita fixed assets investment (unit: million), per capita number of patent application (unit: pieces/ten thousand) and R\&D per capita expenditure (yuan/person) as variables to observe the impact of the supply of public goods and construction of assets, human capital and technology of areas.

\section{Empirical Analysis}

In this paper, Moran's I test is used to detect the existence of spatial autocorrelation in the residual items in regression analysis. The results are shown in Table 1. 
Table 1 Moran's I Spatial Autocorrelation Test Results for Per Capita GDP Growth

\begin{tabular}{lcc}
\hline \hline Year & Moran's I & Z value \\
\hline 2007 & 0.164 & 5.396 \\
2008 & 0.173 & 5.590 \\
2009 & 0.176 & 5.659 \\
2010 & 0.180 & 5.730 \\
2011 & 0.179 & 5.687 \\
2012 & 0.177 & 5.623 \\
2013 & 0.175 & 5.552 \\
2014 & 0.168 & 5.359 \\
\hline \hline
\end{tabular}

Note: Moran's I values are all significant at the 1\% significance level.

From the above Moran's I test, it can be observed that all Moran's I values between 2007 and 2014 are greater than zero and significant at 1\% significance level. It shows that the economic development of various regions is positively correlated in space. Therefore, the spatial econometric model can be used to study the effect of the public goods supply and construction on the regional economy in China.

In order to ensure the robustness of the test results, the following steps are used to screen the above four spatial econometric models. The first step is to establish the SDM model and perform the LM Test and Wald Test on the SDM model data. The test results show that SDM model, SEM model, SLM model and SAC model are all suitable for the panel data selected in this paper. In the second step, individual fixed regression, time fixed regression, and both fixed regression under each model are conducted. The R-square and Loglikelihood under each regression are compared, and the larger the value is, the better the goodness of fit the regression is. In the third step, AIC and BIC tests are performed for each regression in the second step. AIC and BIC are designed to examine the degree of distortion of the processed data compared to the original data. The lower these two values, the more reliable the spatial regression model is. Table 2 shows the analysis of the impact of public goods on regional economic growth using four spatial regression models. In the end, this paper selects both fixed regression of SAC model, which has the best relative regression result and negative AIC and BIC values, to examine the impact of public goods investment on the growth of regional economy. 
Table 2 Empirical Results of Impact of Public Goods on Regional Economic Development

\begin{tabular}{|c|c|c|c|c|c|c|c|}
\hline \multirow{2}{*}{ Variables } & \multirow{2}{*}{$\begin{array}{l}\text { Panel } \\
\text { Data } \\
\text { Fixed } \\
\text { Effect }\end{array}$} & \multicolumn{3}{|c|}{ SEM Model } & \multicolumn{3}{|c|}{ SLM Model } \\
\hline & & Individual & Time & Both & Individual & Time & Both \\
\hline $\begin{array}{l}\text { Public Service Per } \\
\text { Capita Expenditure }\end{array}$ & $\begin{array}{l}-1.8330 \\
(0.9025)\end{array}$ & $\begin{array}{l}-2.0748 \\
(0.7052)\end{array}$ & $\begin{array}{l}-4.8940 \\
(1.2870)\end{array}$ & $\begin{array}{l}-1.9421 \\
(0.6881)\end{array}$ & $\begin{array}{l}-2.6127 \\
(0.7321)\end{array}$ & $\begin{array}{l}-4.7861 \\
(1.3018)\end{array}$ & $\begin{array}{l}-1.9976 \\
(0.6863) \\
\end{array}$ \\
\hline $\begin{array}{l}\text { National Defense } \\
\text { Per Capita } \\
\text { Expenditure }\end{array}$ & $\begin{array}{r}35.4609 \\
(20.9948) \\
\end{array}$ & $\begin{array}{c}16.9477 \\
(16.5393) \\
\end{array}$ & $\begin{array}{l}-33.9860 \\
(29.7878) \\
\end{array}$ & $\begin{array}{r}16.2534 \\
(16.2514) \\
\end{array}$ & $\begin{array}{c}35.4162 \\
(16.8744)\end{array}$ & $\begin{array}{l}-27.0506 \\
(29.4634)\end{array}$ & $\begin{array}{r}17.0633 \\
(16.2207) \\
\end{array}$ \\
\hline $\begin{array}{l}\text { Security Per Capita } \\
\text { Expenditure }\end{array}$ & $\begin{array}{c}2.5372 \\
(3.5988) \\
\end{array}$ & $\begin{array}{l}4.3445 \\
(2.9397)\end{array}$ & $\begin{array}{l}36.0285 \\
(3.7880)\end{array}$ & $\begin{array}{c}4.6595 \\
(2.8345) \\
\end{array}$ & $\begin{array}{c}5.2202 \\
(2.9126)\end{array}$ & $\begin{array}{l}34.7345 \\
(3.8248)\end{array}$ & $\begin{array}{c}4.2638 \\
(2.8647) \\
\end{array}$ \\
\hline $\begin{array}{c}\text { Education Per } \\
\text { Capita Expenditure }\end{array}$ & $\begin{array}{c}4.2963 \\
(0.9256)\end{array}$ & $\begin{array}{c}1.0832 \\
(0.9193)\end{array}$ & $\begin{array}{l}-0.8635 \\
(1.7095)\end{array}$ & $\begin{array}{c}0.2062 \\
(0.8865) \\
\end{array}$ & $\begin{array}{c}1.9652 \\
(0.8009)\end{array}$ & $\begin{array}{l}-0.5810 \\
(1.6888)\end{array}$ & $\begin{array}{c}0.2951 \\
(0.8818)\end{array}$ \\
\hline $\begin{array}{c}\text { Scientific } \\
\text { Construction Per } \\
\text { Capita Expenditure }\end{array}$ & $\begin{array}{r}4.9089 \\
(3.6536)\end{array}$ & $\begin{array}{l}5.5482 \\
(2.7645)\end{array}$ & $\begin{array}{l}29.2312 \\
(3.8936)\end{array}$ & $\begin{array}{c}4.8447 \\
(2.7388)\end{array}$ & $\begin{array}{l}-0.1464 \\
(3.0062)\end{array}$ & $\begin{array}{l}29.6848 \\
(4.0674)\end{array}$ & $\begin{array}{l}5.1829 \\
(2.8139)\end{array}$ \\
\hline $\begin{array}{l}\text { Culture, Sports and } \\
\text { Media Per Capita } \\
\text { Expenditure }\end{array}$ & $\begin{array}{l}-0.7070 \\
(4.3674) \\
\end{array}$ & $\begin{array}{r}9.5591 \\
(3.3815) \\
\end{array}$ & $\begin{array}{r}0.7433 \\
(7.0789) \\
\end{array}$ & $\begin{array}{r}9.0607 \\
(3.3233) \\
\end{array}$ & $\begin{array}{r}3.0310 \\
(3.5424) \\
\end{array}$ & $\begin{array}{r}2.0867 \\
(7.1650) \\
\end{array}$ & $\begin{array}{r}9.9618 \\
(3.3437) \\
\end{array}$ \\
\hline $\begin{array}{c}\text { Social Security } \\
\text { Employment Per } \\
\text { Capita Expenditure }\end{array}$ & $\begin{array}{l}1.5172 \\
(0.8777)\end{array}$ & $\begin{array}{c}1.5181 \\
(0.6446)\end{array}$ & $\begin{array}{l}-1.3633 \\
(0.8992)\end{array}$ & $\begin{array}{r}1.2268 \\
(0.6441)\end{array}$ & $\begin{array}{r}0.8866 \\
(0.7100)\end{array}$ & $\begin{array}{l}-1.3766 \\
(0.8967)\end{array}$ & $\begin{array}{r}1.2926 \\
(0.6373)\end{array}$ \\
\hline $\begin{array}{c}\text { Health Care Per } \\
\text { Capita Expenditure }\end{array}$ & $\begin{array}{c}4.6442 \\
(2.4992)\end{array}$ & $\begin{array}{c}2.5766 \\
(3.0603) \\
\end{array}$ & $\begin{array}{l}-5.9462 \\
(3.9532)\end{array}$ & $\begin{array}{l}-1.8932 \\
(2.6520)\end{array}$ & $\begin{array}{l}-4.1135 \\
(2.2970)\end{array}$ & $\begin{array}{l}-6.6920 \\
(4.0390)\end{array}$ & $\begin{array}{l}-1.7399 \\
(2.7309) \\
\end{array}$ \\
\hline $\begin{array}{c}\text { Environmental } \\
\text { Protection Per } \\
\text { Capita Expenditure }\end{array}$ & $\begin{array}{l}10.3706 \\
(3.0353)\end{array}$ & $\begin{array}{l}8.2474 \\
(2.3009)\end{array}$ & $\begin{array}{l}12.9696 \\
(3.3139)\end{array}$ & $\begin{array}{c}7.1442 \\
(2.2388)\end{array}$ & $\begin{array}{r}8.3395 \\
(2.4532)\end{array}$ & $\begin{array}{l}13.6209 \\
(3.2746)\end{array}$ & $\begin{array}{c}7.4420 \\
(2.2371)\end{array}$ \\
\hline $\begin{array}{c}\text { Urban and Rural } \\
\text { Community Affairs } \\
\text { Per Capita } \\
\text { Expenditure } \\
\end{array}$ & $\begin{array}{l}0.2369 \\
(0.6624)\end{array}$ & $\begin{array}{l}0.9415 \\
(0.5415)\end{array}$ & $(0.8067)$ & $\begin{array}{c}1.6942 \\
(0.5254)\end{array}$ & $(0.5710)$ & $\begin{array}{l}-2.4896 \\
(0.8315)\end{array}$ & $\begin{array}{r}1.5764 \\
(0.5137)\end{array}$ \\
\hline $\begin{array}{c}\text { Agriculture, } \\
\text { Forestry and Water } \\
\text { Affairs Per Capita } \\
\text { Expenditure }\end{array}$ & $\begin{array}{l}-2.1896 \\
(1.3886)\end{array}$ & $\begin{array}{l}-3.5722 \\
(1.1036)\end{array}$ & $\begin{array}{l}-10.1221 \\
(1.3185)\end{array}$ & $\begin{array}{l}-3.8086 \\
(1.0652)\end{array}$ & -2.7184 & $\begin{array}{l}-9.9099 \\
(1.3382)\end{array}$ & $\begin{array}{l}-3.7084 \\
(1.0691)\end{array}$ \\
\hline $\begin{array}{l}\text { Transportation Per } \\
\text { Capita Expenditure }\end{array}$ & $\begin{array}{l}-2.3158 \\
(0.7345) \\
\end{array}$ & $\begin{array}{l}-1.7346 \\
(0.6175) \\
\end{array}$ & $\begin{array}{c}0.1754 \\
(1.0106) \\
\end{array}$ & $\begin{array}{l}-1.0351 \\
(0.5868) \\
\end{array}$ & $\begin{array}{l}-0.8539 \\
(0.6189) \\
\end{array}$ & $\begin{array}{l}-0.0396 \\
(1.0279) \\
\end{array}$ & $\begin{array}{l}-1.2151 \\
(0.5849) \\
\end{array}$ \\
\hline $\begin{array}{c}\text { Education } \\
\text { Population (College } \\
\text { and above) }\end{array}$ & $\begin{array}{c}0.0001 \\
(0.00002) \\
\end{array}$ & $\begin{array}{l}0.00002 \\
(0.00002)\end{array}$ & $\begin{array}{c}0.0001 \\
(0.00002)\end{array}$ & $\begin{array}{l}-0.00001 \\
(0.00002)\end{array}$ & $\begin{array}{c}0.00002 \\
(0.00002)\end{array}$ & $\begin{array}{c}0.0001 \\
(0.00002)\end{array}$ & $\begin{array}{l}-0.00001 \\
(0.00002) \\
\end{array}$ \\
\hline $\begin{array}{l}\text { Regional Per Capita } \\
\text { Investment in Fixed } \\
\text { Assets }\end{array}$ & $\begin{array}{l}0.4577 \\
(0.0400)\end{array}$ & $\begin{array}{l}0.4407 \\
(0.0351)\end{array}$ & $\begin{array}{r}0.8691 \\
(0.0409)\end{array}$ & $\begin{array}{r}0.4061 \\
(0.0338)\end{array}$ & $\begin{array}{l}0.3577 \\
(0.0346)\end{array}$ & $\begin{array}{r}0.8626 \\
(0.0448)\end{array}$ & $\begin{array}{r}0.4074 \\
(0.0336)\end{array}$ \\
\hline $\begin{array}{c}\text { Patent Per Capita } \\
\text { Acceptance }\end{array}$ & $\begin{array}{c}0.0063 \\
(0.0067)\end{array}$ & $\begin{array}{c}0.0070 \\
(0.0050)\end{array}$ & $\begin{array}{c}0.0047 \\
(0.0071)\end{array}$ & $\begin{array}{c}0.0108 \\
(0.0049) \\
\end{array}$ & $\begin{array}{c}0.0125 \\
(0.0054)\end{array}$ & $\begin{array}{c}0.0043 \\
(0.0071)\end{array}$ & $\begin{array}{c}0.0102 \\
(0.0049) \\
\end{array}$ \\
\hline $\begin{array}{l}\text { Regional R\&D Per } \\
\text { Capita Expenditure }\end{array}$ & $\begin{array}{c}0.0012 \\
(0.0001)\end{array}$ & $\begin{array}{c}0.0011 \\
(0.0001)\end{array}$ & $\begin{array}{c}0.0011 \\
(0.0002)\end{array}$ & $\begin{array}{c}0.0011 \\
(0.0001) \\
\end{array}$ & $\begin{array}{c}0.0010 \\
(0.0001)\end{array}$ & $\begin{array}{c}0.0011 \\
(0.0002)\end{array}$ & $\begin{array}{c}0.0011 \\
(0.0001) \\
\end{array}$ \\
\hline $\begin{array}{c}\mathrm{R}^{\wedge} 2 \\
\text { Log-Likelyhood } \\
\end{array}$ & 0.9111 & $\begin{array}{c}0.9309 \\
130.7805 \\
\end{array}$ & $\begin{array}{c}0.9701 \\
-70.7439 \\
\end{array}$ & $\begin{array}{c}0.9308 \\
155.1615 \\
\end{array}$ & $\begin{array}{c}0.9313 \\
154.5979 \\
\end{array}$ & $\begin{array}{c}0.9703 \\
-71.9755 \\
\end{array}$ & $\begin{array}{c}0.8689 \\
123.0623 \\
\end{array}$ \\
\hline
\end{tabular}


Table 3 Empirical Results of Impact of Public Goods on Regional Economic Development

\begin{tabular}{|c|c|c|c|c|c|c|}
\hline \multirow{2}{*}{ Variables } & \multicolumn{3}{|c|}{ SAC Model } & \multicolumn{3}{|c|}{ SDM Model } \\
\hline & Individual & Time & Both & Individual & Time & Both \\
\hline $\begin{array}{l}\text { Public Service Per } \\
\text { Capita Expenditure }\end{array}$ & $\begin{array}{l}-2.1451 \\
(0.7003)\end{array}$ & $\begin{array}{l}-4.8832 \\
(1.2904)\end{array}$ & $\begin{array}{l}-1.9470 \\
(0.6888)\end{array}$ & $\begin{array}{l}-1.9638 \\
(0.6927)\end{array}$ & $\begin{array}{l}-4.8673 \\
(1.1788)\end{array}$ & $\begin{array}{l}-2.2093 \\
(0.7434)\end{array}$ \\
\hline $\begin{array}{l}\text { National Defense Per } \\
\text { Capita Expenditure }\end{array}$ & $\begin{array}{c}20.4123 \\
(16.5011)\end{array}$ & $\begin{array}{l}-33.9865 \\
(29.7915)\end{array}$ & $\begin{array}{c}16.3397 \\
(16.2585)\end{array}$ & $\begin{array}{c}14.5512 \\
(15.8852)\end{array}$ & $\begin{array}{l}-10.2981 \\
(27.6963)\end{array}$ & $\begin{array}{c}10.2940 \\
(15.5133)\end{array}$ \\
\hline $\begin{array}{l}\text { Security Per Capita } \\
\text { Expenditure }\end{array}$ & $\begin{array}{c}4.6115 \\
(2.9135)\end{array}$ & $\begin{array}{l}35.9572 \\
(3.8454)\end{array}$ & $\begin{array}{c}4.5783 \\
(2.8706)\end{array}$ & $\begin{array}{c}4.0340 \\
(2.9164)\end{array}$ & $\begin{array}{l}30.0353 \\
(3.7786)\end{array}$ & $\begin{array}{c}5.6696 \\
(2.8482)\end{array}$ \\
\hline $\begin{array}{l}\text { Education Per Capita } \\
\text { Expenditure }\end{array}$ & $\begin{array}{c}1.0947 \\
(0.8797) \\
\end{array}$ & $\begin{array}{l}-0.8667 \\
(1.7093) \\
\end{array}$ & $\begin{array}{c}0.2077 \\
(0.8866)\end{array}$ & $\begin{array}{c}1.5964 \\
(0.9064) \\
\end{array}$ & $\begin{array}{c}0.6883 \\
(1.6443) \\
\end{array}$ & $\begin{array}{c}1.6623 \\
(0.8811) \\
\end{array}$ \\
\hline $\begin{array}{l}\text { Scientific Construction } \\
\text { Per Capita Expenditure }\end{array}$ & $\begin{array}{c}3.6215 \\
(2.8168) \\
\end{array}$ & $\begin{array}{l}29.3632 \\
(4.0871)\end{array}$ & $\begin{array}{c}4.9832 \\
(2.8490)\end{array}$ & $\begin{array}{c}1.1926 \\
(3.2048)\end{array}$ & $\begin{array}{l}20.0517 \\
(4.8131)\end{array}$ & $\begin{array}{c}2.0815 \\
(3.1107) \\
\end{array}$ \\
\hline $\begin{array}{l}\text { Culture, Sports and } \\
\text { Media Per Capita } \\
\text { Expenditure }\end{array}$ & $\begin{array}{c}8.2380 \\
(3.3556)\end{array}$ & $\begin{array}{c}0.8803 \\
(7.1978)\end{array}$ & $\begin{array}{c}9.2145 \\
(3.4396)\end{array}$ & $\begin{array}{c}1.4510 \\
(3.9400)\end{array}$ & $\begin{array}{c}8.5605 \\
(7.2495)\end{array}$ & $\begin{array}{c}4.7126 \\
(3.7018)\end{array}$ \\
\hline $\begin{array}{c}\text { Social Security } \\
\text { Employment Per Capita } \\
\text { Expenditure }\end{array}$ & $\begin{array}{c}1.3372 \\
(0.6492)\end{array}$ & $\begin{array}{l}-1.3723 \\
(0.9026)\end{array}$ & $\begin{array}{c}1.2349 \\
(0.6455)\end{array}$ & $\begin{array}{l}1.3765 \\
(0.7400)\end{array}$ & $\begin{array}{l}-2.7216 \\
(0.9740)\end{array}$ & $\begin{array}{c}1.4662 \\
(0.7992)\end{array}$ \\
\hline $\begin{array}{l}\text { Health Care Per Capita } \\
\text { Expenditure } \\
\end{array}$ & $\begin{array}{c}0.7427 \\
(2.6300) \\
\end{array}$ & $\begin{array}{l}-5.9906 \\
(3.9813) \\
\end{array}$ & $\begin{array}{l}-2.0045 \\
(2.7287) \\
\end{array}$ & $\begin{array}{l}-1.5521 \\
(2.7872) \\
\end{array}$ & $\begin{array}{l}-4.9439 \\
(3.5628) \\
\end{array}$ & $\begin{array}{l}-3.3324 \\
(2.6248) \\
\end{array}$ \\
\hline $\begin{array}{c}\text { Environmental } \\
\text { Protection Per Capita } \\
\text { Expenditure } \\
\end{array}$ & $\begin{array}{c}8.0106 \\
(2.3017)\end{array}$ & $\begin{array}{l}12.9500 \\
(3.3176)\end{array}$ & $\begin{array}{c}7.1845 \\
(2.2513)\end{array}$ & $\begin{array}{c}6.0080 \\
(2.4894)\end{array}$ & $\begin{array}{l}14.9234 \\
(3.6046)\end{array}$ & $\begin{array}{c}3.7853 \\
(2.5115)\end{array}$ \\
\hline $\begin{array}{c}\text { Urban and Rural } \\
\text { Community Affairs Per } \\
\text { Capita Expenditure } \\
\end{array}$ & $\begin{array}{c}1.2477 \\
(0.5237)\end{array}$ & $\begin{array}{l}-2.4932 \\
(0.8224)\end{array}$ & $\begin{array}{c}1.6842 \\
(0.5285)\end{array}$ & $\begin{array}{l}2.9036 \\
(0.6559)\end{array}$ & $\begin{array}{l}-1.5096 \\
(0.9670)\end{array}$ & $\begin{array}{c}3.0784 \\
(0.6124)\end{array}$ \\
\hline $\begin{array}{l}\text { Agriculture, Forestry } \\
\text { and Water Affairs Per } \\
\text { Capita Expenditure }\end{array}$ & $\begin{array}{l}-3.4220 \\
(1.0979)\end{array}$ & $\begin{array}{l}-10.1089 \\
(1.3252)\end{array}$ & $\begin{array}{l}-3.7930 \\
(1.0691)\end{array}$ & $\begin{array}{l}-2.6268 \\
(1.1424)\end{array}$ & $\begin{array}{l}-6.5979 \\
(1.3844)\end{array}$ & $\begin{array}{l}-3.0332 \\
(1.0618)\end{array}$ \\
\hline $\begin{array}{l}\text { Transportation Per } \\
\text { Capita Expenditure }\end{array}$ & $\begin{array}{l}-1.4653 \\
(0.6070) \\
\end{array}$ & $\begin{array}{c}0.1650 \\
(1.0159) \\
\end{array}$ & $\begin{array}{l}-1.0571 \\
(0.6006)\end{array}$ & $\begin{array}{l}-1.3014 \\
(0.6203) \\
\end{array}$ & $\begin{array}{l}-3.3477 \\
(1.0296)\end{array}$ & $\begin{array}{l}-1.3598 \\
(0.6114) \\
\end{array}$ \\
\hline $\begin{array}{l}\text { Education Population } \\
\text { (College and above) }\end{array}$ & $\begin{array}{c}0.00001 \\
(0.00002)\end{array}$ & $\begin{array}{c}0.00007 \\
(0.00002)\end{array}$ & $\begin{array}{l}-0.00001 \\
(0.00002)\end{array}$ & $\begin{array}{l}-0.00002 \\
(0.00002)\end{array}$ & $\begin{array}{c}0.00005 \\
(0.00002)\end{array}$ & $\begin{array}{l}-0.00003 \\
(0.00002)\end{array}$ \\
\hline $\begin{array}{c}\text { Regional Per Capita } \\
\text { Investment in Fixed } \\
\text { Assets }\end{array}$ & $\begin{array}{c}0.4263 \\
(0.0335)\end{array}$ & $\begin{array}{c}0.8711 \\
(0.0451)\end{array}$ & $\begin{array}{l}0.4059 \\
(0.0338)\end{array}$ & $\begin{array}{l}0.4119 \\
(0.0347)\end{array}$ & $\begin{array}{l}0.8503 \\
(0.0416)\end{array}$ & $\begin{array}{c}0.3720 \\
(0.0357)\end{array}$ \\
\hline $\begin{array}{l}\text { Patent Per Capita } \\
\text { Acceptance }\end{array}$ & $\begin{array}{c}0.0091 \\
(0.0050)\end{array}$ & $\begin{array}{c}0.0047 \\
(0.0071)\end{array}$ & $\begin{array}{c}0.0108 \\
(0.0049)\end{array}$ & $\begin{array}{c}0.0083 \\
(0.0056)\end{array}$ & $\begin{array}{c}0.0213 \\
(0.0078)\end{array}$ & $\begin{array}{c}0.0077 \\
(0.0054)\end{array}$ \\
\hline $\begin{array}{l}\text { Regional R\&D Per } \\
\text { Capita Expenditure }\end{array}$ & $\begin{array}{c}0.0011 \\
(0.0001)\end{array}$ & $\begin{array}{c}0.0011 \\
(0.0002)\end{array}$ & $\begin{array}{c}0.0011 \\
(0.0001)\end{array}$ & $\begin{array}{c}0.0012 \\
(0.0001) \\
\end{array}$ & $\begin{array}{c}0.0012 \\
(0.0002) \\
\end{array}$ & $\begin{array}{c}0.0012 \\
(0.0001)\end{array}$ \\
\hline $\begin{array}{c}\mathrm{R}^{\wedge} 2 \\
\text { Log-Likelyhood }\end{array}$ & $\begin{array}{c}0.895 \\
138.3237\end{array}$ & $\begin{array}{c}0.9701 \\
-70.7354\end{array}$ & $\begin{array}{c}0.9307 \\
155.1821\end{array}$ & $\begin{array}{c}0.891 \\
157.3231\end{array}$ & $\begin{array}{c}0.7406 \\
-47.7086\end{array}$ & $\begin{array}{c}0.0391 \\
177.1744\end{array}$ \\
\hline
\end{tabular}

The SAC model results show that per capita fiscal expenditure on defense has a very large role in promoting the regional economic growth. 1 yuan increase in per capita fiscal expenditure on defense is predicted to increase GDP by 16.3397 yuan. But the standard deviation is 16.2385 , which is a big deviation. The effect of other fiscal expenditure variables on regional economic growth can be roughly divided into three categories. The first group has a significant role in promoting regional economic growth, including 
security per capita expenditure, scientific construction per capita expenditure, cultural and sports and media per capita expenditure, social security, employment per capita expenditure and environmental protection per capita expenditure. The second category still has a positive effect on regional economic development, but the extent is weaker than the first one, such as regional fixed assets investment per capita, the number of regional per capita patents, and the per capita R\&D expenditure per capita. Variables in the third group have no significant effect on promoting regional economic growth. For example, although the population of college education and above has a positive impact on regional economic development, its impact is very weak. Meanwhile, health care per capita expenditure, agriculture, forestry and water affairs per capita expenditure and transportation per capita expenditure have negative effect on the regional economic growth.

In this paper, the cumulative effect of spatial spillover is analyzed by the individual fixed method of SDM model, and the regression results are shown in Table 4. This paper divides each public goods'role in regional economic growth into direct effect, which is the impact of regional economic growth, and indirect effect, which is the impact of economic growth in the surrounding area on the region per se. Direct and indirect effects provide the overall effect of public goods investment. At the same time, because of the significant differences in the degree of regional economic development in China, this paper also calculates the spatial spillover effects in eastern, central and western regions of China separately, and observes the differences. According to the spatial econometric regression results, the spillover effect of public goods investment does exist, which means that investment in local public goods can promote the economy of the surrounding areas. Meanwhile, public goods for the eastern, central and western regions of the economy can bring different effects. For example, local fiscal expenditure in science has a coefficient of 1.1926 for the effect of economic growth in nationwide while for western region it is as high as 5.8138. In addition, the spillover effect of the scientific construction of public goods investment is huge in the central and eastern regions. As for the local fiscal transportation and transportation variables, on the national scale, investment in such public goods have a positive effect on the regional economy, but the effect of this kind of public goods investment on the eastern region is much less than the central region. Some variables even have a negative effect on the economic development in the west. Not only that, investment in public transportation in the eastern and central regions have a catalytic effect on the economic development of the region and the surrounding areas, but it has a negative effect on the economic development of the western region. It means that the investment of 1 yuan for transportation projects is estimated to reduce the GDP of the western region by 3.4 yuan. On the contrary, investing 1 yuan in this type of project in the 
central region is predicted to increase the GDP of the central region by 18.6 yuan.

Table 4 Cumulative Effect of Spatial Spillover

\begin{tabular}{|c|c|c|c|c|c|}
\hline Varibles & Effects & Nationwide & Eastern & Central & Western \\
\hline \multirow{3}{*}{$\begin{array}{l}\text { Public Service Per Capita } \\
\text { Expenditure }\end{array}$} & Direct & -1.9638 & 2.7875 & 4.9059 & 0.398126 \\
\hline & Indirect & 1.2536 & -15.77021 & 2.681574 & 2.699971 \\
\hline & Overall & -0.7102 & -12.982701 & 7.587484 & 3.098097 \\
\hline \multirow{3}{*}{$\begin{array}{l}\text { National Defense Per } \\
\text { Capita Expenditure }\end{array}$} & Direct & 14.5512 & -91.92222 & -185.0688 & 8.616048 \\
\hline & Indirect & -5.2709 & -126.8361 & -498.8939 & -108.1344 \\
\hline & Overall & 9.2804 & -218.75832 & -683.9627 & -99.518352 \\
\hline \multirow{3}{*}{$\begin{array}{l}\text { Security Per Capita } \\
\text { Expenditure }\end{array}$} & Direct & 4.0340 & 20.28089 & 44.40509 & -3.755061 \\
\hline & Indirect & 15.9135 & 120.7274 & -10.65144 & 35.21829 \\
\hline & Overall & 19.9475 & 141.00829 & 33.75365 & 31.463229 \\
\hline \multirow{3}{*}{$\begin{array}{l}\text { Education Per Capita } \\
\text { Expenditure }\end{array}$} & Direct & 1.5964 & -2.136185 & 3.508447 & 1.805388 \\
\hline & Indirect & 5.2268 & -10.77213 & -5.572587 & 5.108408 \\
\hline & Overall & 6.8232 & -12.908315 & -2.06414 & 6.913796 \\
\hline \multirow{3}{*}{$\begin{array}{l}\text { Scientific Construction Per } \\
\text { Capita Expenditure }\end{array}$} & Direct & & 1.435931 & 1.679417 & 5.813888 \\
\hline & Indirect & -7.2905 & 1.120485 & 36.0907 & 107.8262 \\
\hline & Overall & -6.0979 & 2.556416 & 37.770117 & 113.640088 \\
\hline \multirow{3}{*}{$\begin{array}{l}\text { Culture, Sports and Media } \\
\text { Per Capita Expenditure }\end{array}$} & Direct & 1.4510 & 5.988217 & 1.842277 & -0.9981896 \\
\hline & Indirect & -119.0075 & -3.421864 & -90.06767 & -53.78335 \\
\hline & Overall & -117.5565 & 2.566353 & -88.225393 & -54.7815396 \\
\hline Social Security & Direct & 1.376457 & 1.374291 & 5.099331 & -0.2744515 \\
\hline \multirow{2}{*}{$\begin{array}{c}\text { Employment Per Capita } \\
\text { Expenditure }\end{array}$} & Indirect & 6.83266 & -4.246854 & 17.08377 & -9.239022 \\
\hline & Overall & 8.209117 & -2.872563 & 22.183101 & -9.5134735 \\
\hline \multirow{3}{*}{$\begin{array}{c}\text { Health Care Per Capita } \\
\text { Expenditure }\end{array}$} & Direct & -1.552061 & -4.75555 & -3.586805 & 8187 \\
\hline & Indirect & -3.404269 & -6.991641 & -49.32734 & 48.36548 \\
\hline & Overall & -4.95633 & -11.747191 & -52.914145 & 51.193667 \\
\hline \multirow{3}{*}{$\begin{array}{l}\text { Environmental Protection } \\
\text { Per Capita Expenditure }\end{array}$} & Dir & 34 & -0.9872365 & 17.3 & 1474 \\
\hline & Indirect & -11.94168 & -70.85889 & 81.4872 & 22.76388 \\
\hline & Overall & -5.933646 & -71.8461265 & 98.85572 & 25.388354 \\
\hline \multirow{3}{*}{$\begin{array}{c}\text { Urban and Rural } \\
\text { Community Affairs Per } \\
\text { Capita Expenditure }\end{array}$} & Direct & 2.903614 & 5.179388 & -0.3539302 & 2.800357 \\
\hline & Indirect & 7.165154 & 8.291147 & -11.19355 & -0.5897057 \\
\hline & Overall & 10.068768 & 13.470535 & -11.5474802 & 2.2106513 \\
\hline \multirow{3}{*}{$\begin{array}{c}\text { Agriculture, Forestry and } \\
\text { Water Affairs Per Capita } \\
\text { Expenditure }\end{array}$} & Direct & -2.626838 & -0.6787048 & -3.68601 & 0.0590279 \\
\hline & Indirect & 0.9275867 & -0.4126099 & -2.800778 & -10.47735 \\
\hline & Overall & -1.6992513 & -1.0913147 & -6.486788 & -10.4183221 \\
\hline \multirow{3}{*}{$\begin{array}{c}\text { Transportation Per Capita } \\
\text { Expenditure }\end{array}$} & Direct & -1.301448 & 1.588427 & 0.2580207 & -1.916691 \\
\hline & Indirect & 3.876045 & 2.964007 & 18.37794 & -1.496642 \\
\hline & Overall & 2.574597 & 4.552434 & 18.6359607 & -3.413333 \\
\hline \multirow{3}{*}{$\begin{array}{c}\text { Regional Education } \\
\text { Population (College and } \\
\text { above) }\end{array}$} & Direct & -0.000019 & 5.59E-06 & 0.0000879 & -0.0001185 \\
\hline & Indirect & $3.71 \mathrm{E}-06$ & 0.000116 & 0.0003292 & -0.0002159 \\
\hline & Overall & -0.00001529 & 0.00012159 & 0.0004171 & -0.0003344 \\
\hline \multirow{3}{*}{$\begin{array}{c}\text { Regional Per Capita } \\
\text { Investment in Fixed Assets }\end{array}$} & Direct & 0.4119061 & 0.2760547 & 0.1310091 & 0.4018853 \\
\hline & Indirect & -0.2146719 & -0.097863 & 0.0133174 & -1.15464 \\
\hline & Overall & 0.1972342 & 0.1781917 & 0.1443265 & -0.7527547 \\
\hline \multirow{3}{*}{$\begin{array}{l}\text { Patent Per capita acceptance } \\
\text { in the region }\end{array}$} & Direct & 0.0083046 & 0.010792 & 0.0016046 & 0.1040151 \\
\hline & Indirect & 0.0258886 & 0.0527276 & 0.0130937 & -0.003978 \\
\hline & Overall & 0.0341932 & 0.0635196 & 0.0146983 & 0.1000371 \\
\hline \multirow{3}{*}{$\begin{array}{c}\text { Regional R\&D Per Capita } \\
\text { Expenditure }\end{array}$} & Direct & 0.0012102 & 0.0009416 & 0.0027562 & 0.0003189 \\
\hline & Indirect & -0.0006654 & -0.0004786 & 0.0025018 & 0.0004634 \\
\hline & Overall & 0.0005448 & 0.000463 & 0.005258 & 0.0007823 \\
\hline
\end{tabular}




\section{Conclusion and Policy Implications}

This paper selects the statistical data of public goods and regional economic conditions in 31 provinces in China from 2007 to 2014, and comprehensively compares the impact of public goods on the economic development of the region under the four spatial econometric regression models, and compares the influence of different types of public goods to regional economic development. The influence is divided into direct effect, indirect effect and overall effect. The research also analyzes the spatial spillover effect of public goods investment construction on regional economic development. The results show that: firstly, there is a significant positive correlation between investment in public goods and regional economic development. Among them, local fiscal security expenditures and local fiscal environmental protection expenditures have the largest contribution. Secondly, the investment in local public goods has a positive impact on the economic growth in the surrounding areas. Some of these public goods can provide even more economic benefits to the surrounding areas.

Judging from the current situation in China, the gap between the rich and the poor in all regions is still very significant compared with that of developed countries, and the level of investment in public goods in all regions is also very different. According to the conclusions, this paper puts forward the following suggestions for the investment supply of public goods in China.

First, insist on the development and construction of public goods, especially the expenditure on public financial goods. According to the empirical research results of this paper, the majority of the public goods have played a role in promoting the regional economic development in China. The development and construction of public goods cannot only speed up the economic development and promote the balanced development of different regions, but also provide convenience to people and share the fruits of economic development as well. The "13th Five-Year Plan for the National Economy and Social Development of the People's Republic of China" also puts forward the principle of continuing to vigorously develop the construction of public goods services. Chinese government should give it full attention and continue to carry out this policy.

Second, consider regional differences and optimize local public goods development. The public goods of the same category have different effects on the economies of different regions, and their spatial spillover effects also change due to changes in the region. Therefore, when investing in the development of public goods in the region, it is necessary to choose the construction focus in light of the actual situation in the region so as to achieve greater investment returns and bring about greater driving effects on the economic growth of the region and its surrounding areas. 
Third, the government should play a leading role in overall planning. Public product service investment is one of the important methods for government intervention in the economy. Through reasonable distribution of local public investment, the government can play a critical and guiding role in local economic development, economic restructuring, industrial structure improvement, social fair distribution, and people's livelihood improvement. Therefore, only if the Chinese government maintains its leading role in the development of public goods investment can it maximize the benefits that public goods bring to economic development, and at the same time eliminate some of the social hidden dangers brought about by the rapid economic development.

Fourth, promote the coordinated development of the regional economy in many aspects in accordance with the actual needs of the region. When choosing investment in public goods, local governments need to consider various factors such as regional development and the living needs of people in the region. It is not suitable to focus on the development of public goods that can bring about the greatest boost to regional economic growth based solely on the results of data analysis. If the government only pay attention to the increase in the total economic output of the region and neglect the needs of other parties, it may encounter unexpected obstacles. The purpose of economic development is to benefit the people, and the measures to develop the regional economy are also diverse and not limited to public goods investment. The government must comprehensively consider the actual needs of the region, coordinate the promotion of regional diversified economic development, and seek balanced development so as to ensure the sustainable development of Chinese economy.

\section{References:}

1. Li, Z., Liu, X. Y. 2012, 'Spillover, Public Goods, and Economic Growth: Empirical Tests and Decomposition of Effects Based on Spatial Panel Models', Statistics and Information Forum, 27, 10, pp. 57-63.

2. Robert E. Lucas, Jr. 1988, 'On the Mechanics of Economic Development', Journal of Monetary Economics, 22, pp. 3-42.

3. Deno K T. 1988, 'The Effect of Public Capital on U.S. Manufacturing Activity: 1970 to 1978'. Southern Economic Journal, 55, 2, pp. 400.

4. Liu, Y. 2010, 'Transportation Infrastructure Investment, Regional Economic Growth and Spatial Overflow Effect: Analysis of Panel Data Based on Highway and Water Transportation', Industrial Economy of China, 12, pp. 37-46. 
5. Luo, L. Y., Liu, L. 2015, 'Spatial Spillover Effect of Public Product Supply on Regional Economy_-Discussion on SDM Model Based on Panel Data', Hu Xiang Forum, 4, 163, pp. 89-95.

6. Arrow, Kenneth J. and Kurz, Mordecai. 1970, 'Optimal Growth with Irreversible Investment in a Ramsey Model', Econometrica, 38, 2, pp. 331-344.

7. Aschauer D. A. 1989, 'Is Public Expenditure Productive?' Journal of Monetary Economics, 23, 2, pp. 177-200.

8. Boldeanu F T, Ion M S. 2015, 'The Impact of Fiscal Policy on Economic Growth in the Founding Countries of the European Union', Revista Economica.

9. Butkiewicz, James L and Yanikkaya, Halit. 2011, 'Institutions and the Impact of Government Spending on Growth', Journal of Applied Economics, 14, 2, pp. 319-341.

10. Liu, J. Y. 2013, 'The Impact of Government Public Expenditure on the Coordinated Development of Regional Economy: Empirical Evidence Based on Provincial Panel Data in China', Exploration of Economic Issues, 3, pp. 49-55. 